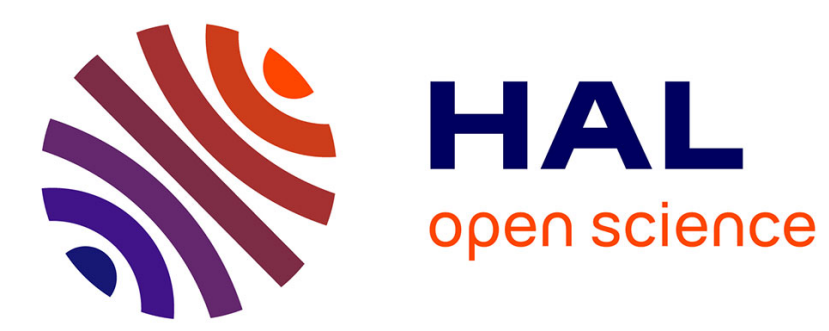

\title{
X-band and K-band low-phase-noise VCOs using SiGe BiCMOS technology
}

\author{
Jean-Guy Tartarin, K.W. W Wong
}

\section{To cite this version:}

Jean-Guy Tartarin, K.W. W Wong. X-band and K-band low-phase-noise VCOs using SiGe BiCMOS technology. Semiconductor Science and Technology, 2006, 22 (1), pp.S195-S199. 10.1088/02681242/22/1/S46 . hal-01343971

\section{HAL Id: hal-01343971 \\ https://hal.science/hal-01343971}

Submitted on 22 May 2018

HAL is a multi-disciplinary open access archive for the deposit and dissemination of scientific research documents, whether they are published or not. The documents may come from teaching and research institutions in France or abroad, or from public or private research centers.
L'archive ouverte pluridisciplinaire HAL, est destinée au dépôt et à la diffusion de documents scientifiques de niveau recherche, publiés ou non, émanant des établissements d'enseignement et de recherche français ou étrangers, des laboratoires publics ou privés. 


\title{
X-band and K-band low-phase-noise VCOs using SiGe BiCMOS technology.
}

\author{
J.G. Tartarin ${ }^{1}$, K.W. Wong ${ }^{2}$. \\ ${ }^{1}$ Paul Sabatier University \& LAAS-CNRS, 7 av. Du Colonel Roche, 31.077, Toulouse cedex 4 France. \\ ${ }^{2}$ Institute of Microelectronics, 11 science Park road, Singapore science park II, Singapore 117685 \\ Phone number : +33(0)5.61.33.64.56 \\ E-mail : tartarin@laas.fr
}

\begin{abstract}
From the past two decades, SiGe transistors have been identified as excellent candidates to match requirements both for high purity signal generation and high frequency applications. Moreover, SiGe BiCMOS technology takes benefit of CMOS devices that allow high integration levels for Monolithic Microwave Integrated Circuits (MMIC) Phase Locked Loop (PLL) systems. This work focuses on the only analog circuit of a PLL: the Voltage Controlled Oscillator (VCO) is one of the key stone of a transceiver, because its stability mainly influences the data rate of the channel. X-band and K-band VCOs are investigated. Design rules are thus compared for two different topologies in the X-band. K-band VCO is also designed to assess the technology behavior for higher frequencies. State of the art results are obtained and compared with the best published performances to date. New expressions of Figures Of Merit (FOM) are proposed to provide an accurate comparison between designs featuring scattered performances.
\end{abstract}

\section{Introduction}

BiCMOS SiGe technologies offer great opportunities due to the high integration level with both analog and digital circuits operating at high frequencies, and also base-band modules matched to modern telecommunication systems' requirements. Moreover, the high performance (low noise, high operating frequency,...) associated with the relative low-cost gives advantage to such technologies in comparison with III-V foundries. The low frequency flicker noise (1/f) of SiGe Heteronjunction Bipolar Transistors (HBT) allows the design of MMIC voltage controlled oscillators (VCO) featuring excellent phase-noise: we present different VCO designs manufactured using the STMicroelectronics BiCMOS $0.35 \mu \mathrm{m}$ and $0.25 \mu \mathrm{m}$ processes for X-band and K-band applications.

The paper is divided in three main sections. In the first part is presented a comparison between the reflection architecture (negative resistance) and the parallel feedback architecture operating in X-band. The second section presents the push-push differential oscillator in K-band. The last section proposes a comparison of this work with state of the art MMIC circuits: a new figure of merit (FOM) is introduced.

\section{X-band VCO: reflection / parallel topologies}

\subsection{HBT optimum biasing.}

As the phase noise of an oscillator close to the carrier is mainly determined by the conversion of the transistor's low frequency noise sources through its non-linear elements, $V_{E B}$ and $V_{C E}$ non linearities have been considered for bipolar devices. From previous work [1], it has been found that emitter-base $V_{\mathrm{EB}}$ fluctuations mainly influence the phase noise.

The device's low frequency noise is investigated with direct measurements of the input and output noise current generators. The input noise current generator (referred to as its spectral noise current density $\mathrm{S}_{\mathrm{IB}}$ ) can be expressed as:

$$
S_{I B}=2 q I_{B}+B_{1 / f} \cdot \frac{I_{B}^{2}}{A_{E} \cdot f}
$$

Where $I_{B}$ represents the static base current, $A_{E}$ the emitter area $\left(\mu \mathrm{m}^{2}\right)$, and $B_{1 / \mathrm{f}}$ a coefficient related to the structural quality of the devices $\left(\mathrm{B}_{1 / \mathrm{f}}=4.10^{-9}\right.$ for our devices, close to [2][3]). The noise floor contribution appears for 
frequencies between $1 \mathrm{kHz}$ and $10 \mathrm{kHz}$ depending on the biasing and transistor's size.

Simulations are performed using ADS software (developed by Agilent), and the optimum biasing quiescent point is determined both for the base current $\mathrm{I}_{\mathrm{B}}$ and collector voltage $\mathrm{V}_{\mathrm{CE}}$ using a specific method based on the pushing factor simulation [4]. As transistor LF noise is converted around the carrier, our optimization goal is to reduce the phase conversion coefficient $\mathrm{K}_{\phi}$ of the amplifier (mainly due to a formulation as given in equation 2), keeping enough gain to permit the oscillation (even if the quality factor of the resonating tank is expected to be low for a MMIC design).

$$
K_{\Phi}=\frac{\Delta \Phi}{\Delta V_{E B}}
$$

$\mathrm{K}_{\Phi}$ represents the phase sensitivity to low frequency fluctuations of the intrinsic emitter-base control voltage $\mathrm{V}_{\mathrm{EB}}$. This study is thus equivalent to the optimization of the pushing factor $K_{P}$ on the EB junction [4]. Excellent agreement is found in comparison with the usual residual phase-noise conversion versus the biasing of the transistor (figure 1).

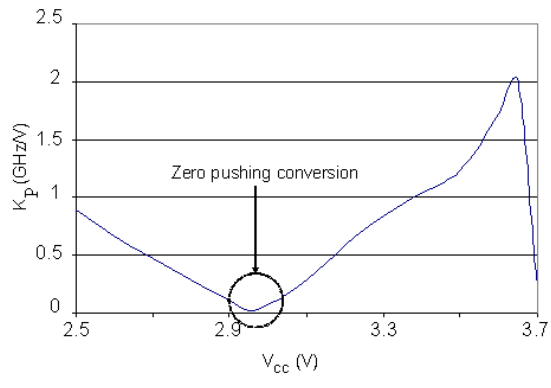

(a) pushing factor

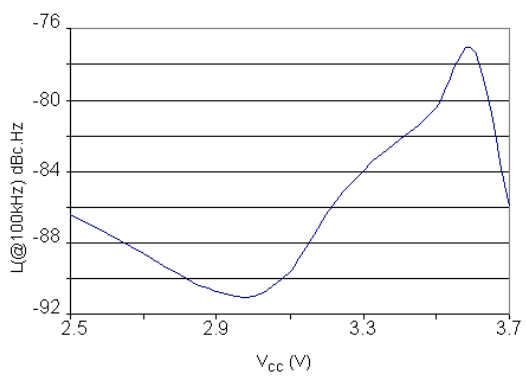

(b) phase noise

Figure 1a-1b pushing conversion factor (a) and phase noise (b) simulations versus biasing voltage $\mathrm{V}_{\mathrm{CC}}$ for the negative resistance $\mathrm{VCO}$.

The BiCMOS6G from ST-Microelectronics is used for the designs in X-band.

\subsection{X-band VCOs performances.}

Two VCOs are designed in X-band for a comparison versus the circuit's topology (reflection and parallel architectures). The two architectures are represented in figure 2, where the resonator is designed to improve simultaneously the quality factor and the tuning range.

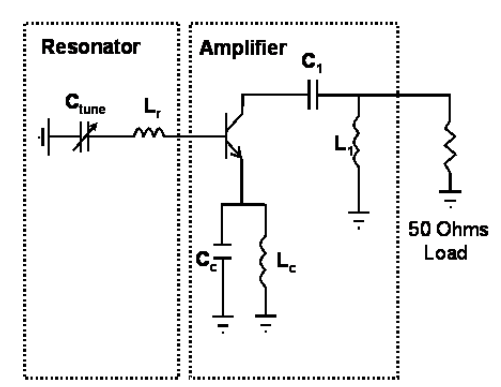

(a) reflection architecture

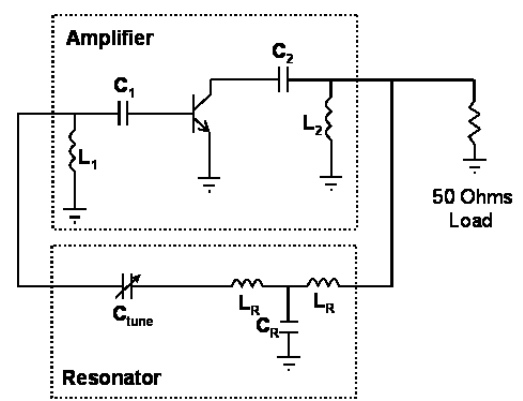

(b) parallel architecture

Figure $2 \mathrm{a}-2 \mathrm{~b}$ electrical schematics of the X-band VCOs using reflection (a) and parallel (b) topologies.

Each circuit makes use of the same HBT device (as the area of the transistor strongly affects the $1 / \mathrm{f}$ noise level) for an easier comparison of the topology's impact on the phase noise: it thus provides the circuit designer with useful criteria to sort out the fitted topology to a specific design.

\subsubsection{Measurement of the X-band reflection $V C O$}

Figure 3 presents the microphotograph of the reflection oscillator (the chip size is $1.4 \times 1 \mathrm{~mm}^{2}$ ). The reflection topology benefits from a higher frequency range for the negative resistance, and a higher loaded quality factor of its resonator in comparison with the parallel feedback topology. The serial feedback circuit measured at $11 \mathrm{GHz}$ features a 
phase-noise of -95 dBc/Hz @ $100 \mathrm{kHz}$ from the carrier as shown in figure 4 (see other performances in table I.).

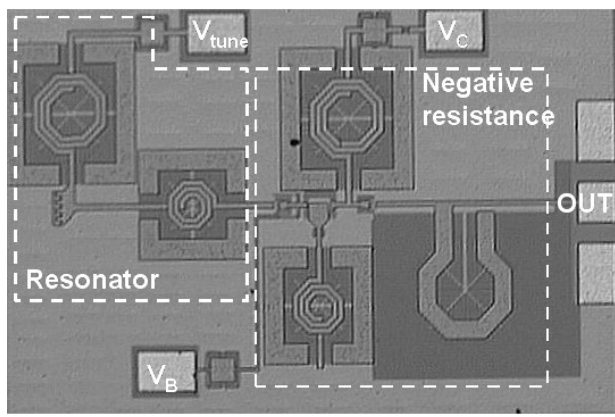

Figure 3 Microphotograph of the X-band reflection VCO

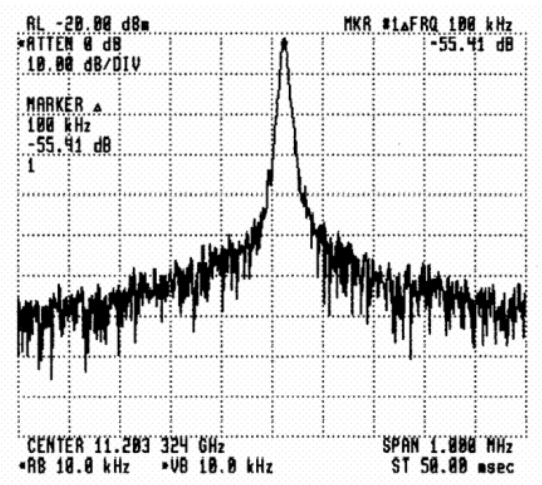

Figure 4 phase noise of the reflection oscillator measured at $-95 \mathrm{dBc} / \mathrm{Hz} @ 100 \mathrm{kHz}$ from the $11 \mathrm{GHz}$ carrier.

\subsubsection{Measurement of the X-band parallel VCO}

A microphotograph of the parallel feedback VCO chip is given in figure 5 (the chip size is $1.2 \times 1 \mathrm{~mm}^{2}$ ).

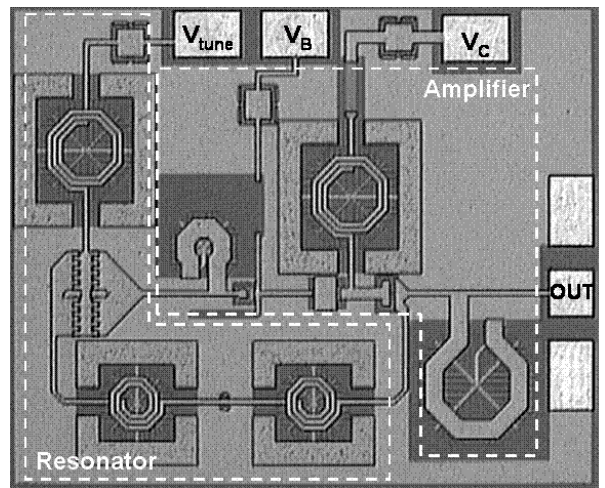

Figure 5 Microphotograph of the X-band parallel feedback VCO

The oscillator features an oscillation frequency of $11 \mathrm{GHz}$ for a $0 \mathrm{~V}$ control voltage and the frequency tuning is about $500 \mathrm{MHz}$ wide for a control voltage from 0 to $3.6 \mathrm{~V}$. On wafer and packaged characterization have been performed for the phase noise measurements. For spectral purity characterization, home made phase noise measurement set-up has been used. This bench is a delay line discriminator, which uses a cross-correlation technique: this measurement technique reaches a noise floor of $-145 \mathrm{dBc} / \mathrm{Hz}$ at $100 \mathrm{kHz}$ offset frequency (X-band). The measured parallel VCO phase noise has been found to be about $-85 \mathrm{dBc} / \mathrm{Hz}$ at $100 \mathrm{kHz}$ offset as shown in figure 6 for a $3.3 \mathrm{~V}$ bias voltage. The cutoff frequency between the $1 / \mathrm{f}^{3}$ and $1 / \mathrm{f}^{2}$ phase noise (respectively related to $1 / \mathrm{f}$ noise conversion, and white noise conversion) is measured at $1.3 \mathrm{kHz}$ (the simulation gives $\mathrm{f}_{\mathrm{c}}=2 \mathrm{kHz}$ from low frequency noise spectrum). 


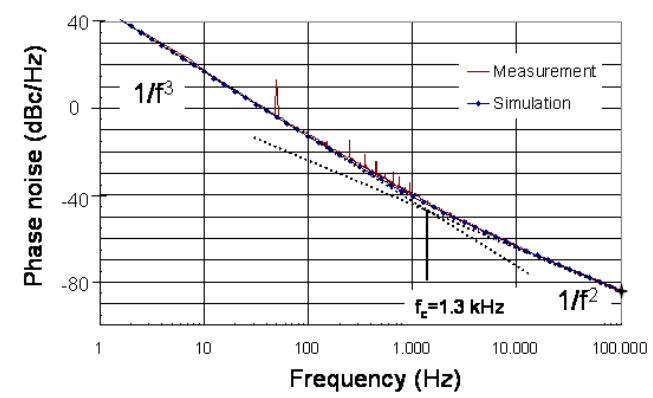

Figure $6 \quad$ Measured and simulated phase noise versus frequency offset (feedback VCO at $\mathrm{V}_{\text {tune }}=0 \mathrm{~V}$ )

The phase noise variation with the transistor biasing is given for two different frequencies from the carrier in figure 7: these two frequencies are associated with two different phase noise behavior featuring $1 / \mathrm{f}^{2}$ and $1 / \mathrm{f}^{3}$ frequency dependency (figure 6). At $100 \mathrm{kHz}$ from the carrier, the improvement of phase noise with a higher biased transistor is obvious. This is correlated to the improvement of the pushing factor $K_{P}$ described in the previous section.

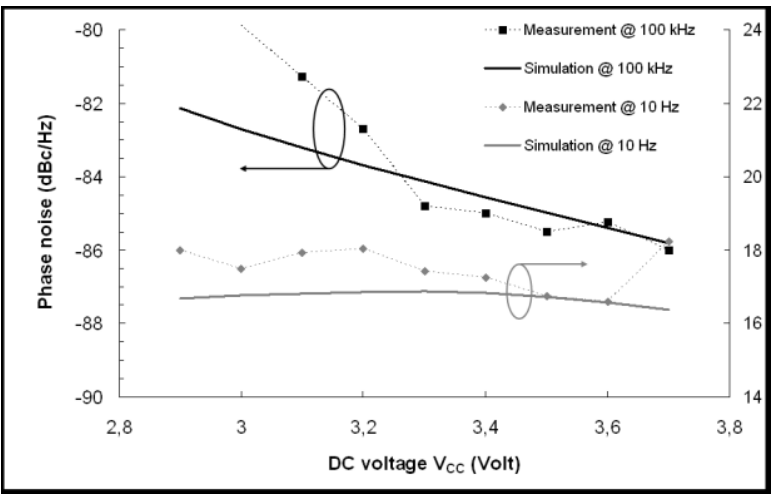

Figure $7 \quad$ Measured and simulated phase noise performance at $10 \mathrm{~Hz}$ and $100 \mathrm{kHz}$ offset versus biasing voltage $\mathrm{V}_{\mathrm{CC}}$, with $\mathrm{I}_{\mathrm{B}-\mathrm{DC}} \approx \mathrm{V}_{\mathrm{CC}} / \mathrm{R}_{\mathrm{B}}$ and $\mathrm{R}_{\mathrm{B}}=14 \mathrm{k} \Omega$

However, at $10 \mathrm{~Hz}$ the phase noise is attributed to the $1 / \mathrm{f}$ low frequency noise conversion source and also to the previous $\mathrm{K}_{\mathrm{p}}$ conversion. The converted $1 / \mathrm{f}$ source is known to be proportional to the base current, whereas the $\mathrm{K}_{\mathrm{P}}$ factor decreases with the base current. This results in a quasi constant phase noise level with $\mathrm{V}_{\mathrm{CC}}$ at $10 \mathrm{~Hz}$ from the carrier.

\section{K-band VCO: differential push-push topology}

A Colpitts differential push-push VCO is designed with the BiCMOS7RF $(0.25 \mu \mathrm{m})$ process from ST Microelectronics. A microphotograph of the K-band VCO is given in figure 8.

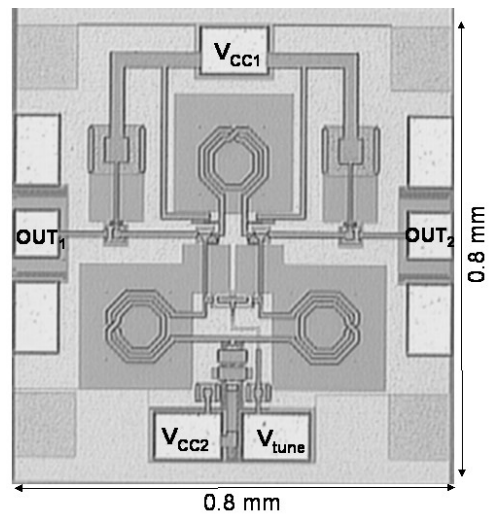

Figure 8 Microphotograph of the K-band VCO. 
The two balanced architectures from figure 9 and figure 10 take advantage from the immunity versus electromagnetic interferences, from the supply bias decoupling (not represented for the bases of the transistors in figure 10), and from the $3 \mathrm{~dB}$ enhancement of the output power. The advantages over usually used topologies also address the phase noise improvement, and negative resistance broadness. These two main topologies featuring anti-phase output signals are the cross-coupled VCO (figure 9) and the push-push VCO [5] (figure 10). In the cross-coupled oscillator scheme (figure 9), each collector node is connected to the base of the other transistor, introducing additive delay time and reducing the operating frequency over that of figure 10. This leads to an adjustment of the transistor sizing to a smaller surface (higher low frequency noise), so that the transistor choice is now closely bound to the architecture, increasing the design complexity. On the contrary, the architecture of figure 10 does not introduce such a delay time, and allows one to operate with higher base area, and lower low frequency noise for a given oscillation frequency.
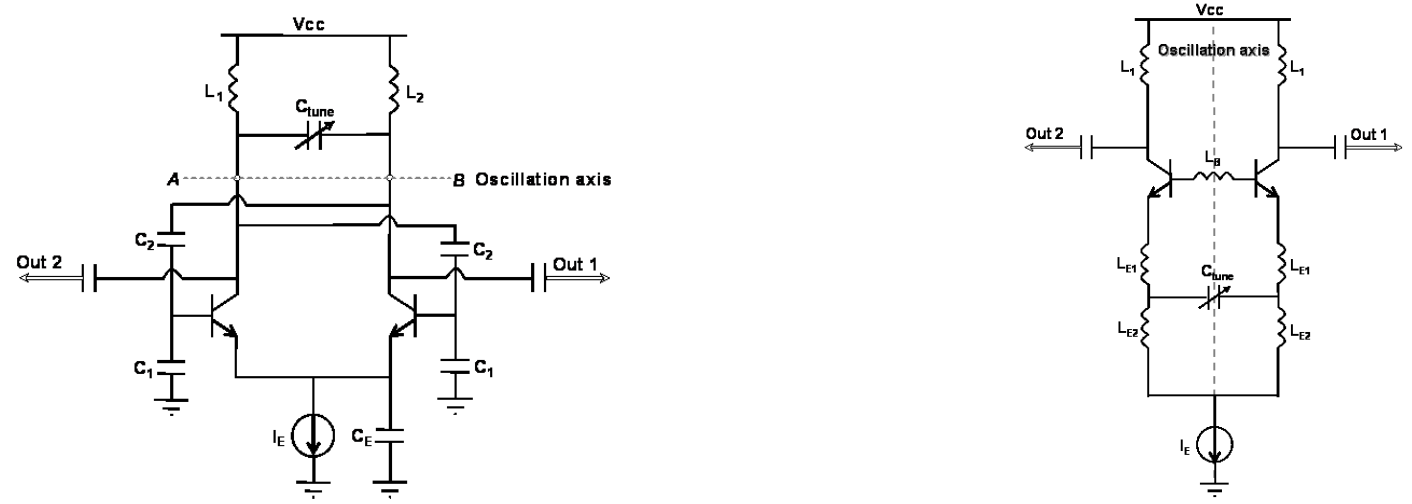

Figure 9 Cross-coupled topology

Figure 10 This work's balanced push-push topology, based on [5]

Moreover, the L-C resonator on figure 9 is heavily loaded by the oscillator output, reducing the loaded quality factor $\left(\mathrm{Q}_{\mathrm{L}}\right)$. In figure 10, the L-C tank is connected to the emitter, and the serial feedback can be adjusted both for single-side-band (SSB) noise reduction, and for improved loaded L-C network quality factor.

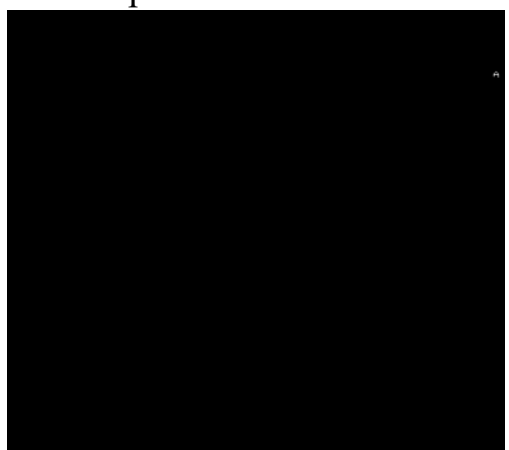

Figure 11 Measured spectrum at $19 \mathrm{GHz}$ featuring phase noise of $-90 \mathrm{dBc} / \mathrm{Hz}(@ 100 \mathrm{kHz})$ and -4 dBm output power.

The phase noise is measured with a spectrum analyzer (figure 11, on wafer measurements). This VCO can be used on a single output (single ended when the second output is terminated with a $50 \Omega$ load), or in a differential configuration according to the circuit to which it is connected. The K-band push-push differential oscillator is measured with a single-ended phase noise of $-90 \mathrm{dBc} / \mathrm{Hz}$ (@100 kHz from the $19 \mathrm{GHz}$ carrier).

\section{Comparison versus state of the art SiGe MMIC using a new FOM expression}

From Table I, the push-push VCO phase noise performance is among the best published results: in bold are the best results using the Leeson's formula (phase noise), the usual FOM (equation 3), or this paper's FOM Pout-stune $_{\text {(equation } 4) .}$

The figure of merit using the 'usual' FOM (equation 3) is -183 for the negative resistance based VCO (section 2), whereas a 'usual' FOM of -168 is obtained for the parallel oscillator (section 2). 


$$
\begin{gathered}
\text { 'usual' FOM }=L\left(f_{m}\right)-20 \log \left[\frac{f_{o}}{f_{m}}\right]+10 \log \left(P_{\text {diss }}\right) \\
\text { FOM Pout-Dtune } \\
=L\left(f_{m}\right)-20 \log \left[\frac{f_{o}}{f_{m}}\right]+10 \log \left(P_{\text {diss }}\right)-10 \log \left(P_{\text {Pout }}\right)-\Delta \text { tune }(\%)
\end{gathered}
$$

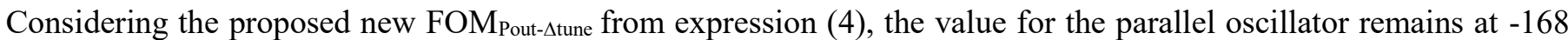
and the reflection topology new figure of merit is -189 . These performances are reported in the table I below, and compared favorably (using the phase-noise Leeson's formula, and other FOMs figures) with the best SiGe based

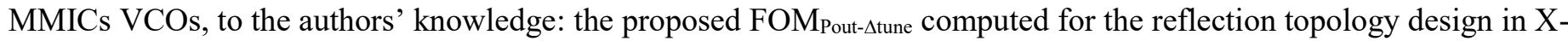
band is very close to the best results published by Li in [5]. The 'usual' FOM in [5] is impressively improved when

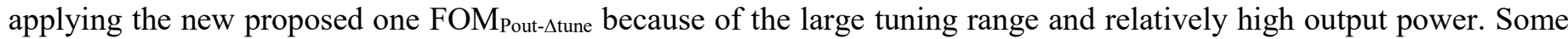

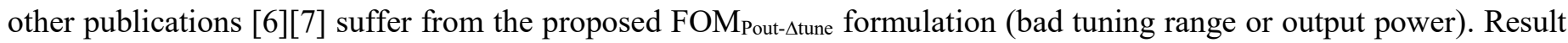
from [8] is very close to that of our X-band reflection topology result (figure 3) for the Leeson's phase noise expression but the lower performances on the tuning range and output power is not favorable for the other FOMs expressions.

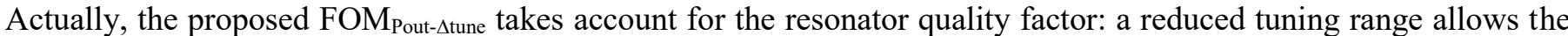
use of inductors featuring higher quality factor, so the phase noise improves. Moreover, a lower DC power can also improve the 'usual' FOM (according to the $-10 \cdot \log \left(\mathrm{P}_{\mathrm{DC}}\right)$ correction), but needs a high gain buffer stage (DC consuming). For the two last reasons, the new FOM Pout- $\Delta$ tune $_{\text {can }}$ be more efficient than the 'usual' FOM to compare VCOs.

\begin{tabular}{|lccccccc|}
\hline \multicolumn{1}{|c}{ Reference } & {$[5]$} & {$[6]$} & {$[7]$} & {$[8]$} & \multicolumn{3}{c|}{ This work } \\
\cline { 6 - 9 } & & & & & $\begin{array}{c}\text { VCO } \\
\text { Fig. 3 }\end{array}$ & $\begin{array}{c}\text { VCO } \\
\text { Fig. 4 }\end{array}$ & $\begin{array}{c}\text { VCO } \\
\text { Fig. 8 }\end{array}$ \\
\hline $\begin{array}{l}\text { Oscillation } \\
\text { frequency (GHz) }\end{array}$ & 46 & 36.8 & 33 & 21.5 & 11.2 & 11 & 19 \\
\hline $\begin{array}{l}\text { Frequency tuning } \\
(\%)\end{array}$ & 26 & 6.8 & 2.2 & 5 & 11 & 4.5 & 12 \\
\hline Pout (dBm) & -4 & -14 & -17 & -6 & -5 & -4 & -6 \\
\hline $\begin{array}{l}\text { Phase noise } \\
\text { MHz) }\end{array}$ & -110 & -99 & -99 & -113 & -115 & -103 & -110 \\
\hline 'Usual' FOM & -179 & -171 & -185 & -179 & -183 & -168 & -176 \\
\hline FOM & -201 & -164 & -170 & -178 & -189 & -168 & -182 \\
\hline
\end{tabular}

Table 1. Best published Low-Phase Noise VCO's Figures Of Merit (FOM) of SiGe MMIC

\section{Conclusions}

X-band and K-band VCOs MMIC using BiCMOS processes have been presented. Excellent low-phase noise performances are obtained thanks to a matched design procedure based on the transistor size and biasing conditions. A comparison of architecture (X-band work) is also proposed for circuit designers. Lastly, a new figure of merit is introduced to better account of additive parameters such as the tuning frequency range and the output power.

\section{Acknowledgements}

The authors wish to acknowledge ST Microelectronics, Crolles, France, for circuit manufacturing, G. Cibiel for residual phase noise measurements, O. Llopis and É. Tournier for fruitful discussions and software assistance.

\section{References}

[1] Llopis O. et al., Annals of Telecommunications, Vol.51, №3-4, pp.121-129, 1996

[2] Jouan et al., IEEE Trans. Electron Devices, 1999, Vol. 46, pp 1525-1531.

[3] Bary L. et al., IEEE MTT-S Int. Microwave Symposium, vol. 1, June 2002, pp. 275-278.

[4] Wong K. W. et al. , IEEE Radio Frequency Integrated Circuits Symp., Dig. vol. 1, June 1997, pp. 255-258.

[5] Li H. et al., IEEE Journal of Solid State Circuits., vol. 38, No. 2, February 2003, pp. 184-191.

[6] Veenstra H. et al., IEEE International Solid-State Circuits Conference, Vol. 1, February 2004, session 21.

[7] Chen Y. J. et al., IEEE Microwave Theory and Techniques Symp., Vol. 53, No. 5, May 2005, pp. 1672-1681.

[8] Bao M. et al., IEEE Journal of Solid-State Circuits, Vol. 39, No. 8, August 2004, pp. 1352-1355. 